\title{
Colloidal metal oxide nanocrystal catalysis by sustained chemically driven
}

\section{ligand displacement}

\author{
Jonathan De Roo ${ }^{1,2,3^{*}}$, Isabel Van Driessche ${ }^{1}$, José C. Martins ${ }^{2}$, Zeger Hens ${ }^{3,4^{*}}$ \\ ${ }^{1}$ Sol-gel Center for Research on Inorganic Powders and Thin films Synthesis, Ghent University, \\ Gent, Belgium \\ ${ }^{2}$ NMR and Structure Analysis Unit, Ghent University, Gent, Belgium \\ ${ }^{3}$ Physics and Chemistry of Nanostructures group, Ghent University, Gent, Belgium \\ ${ }^{4}$ Center for Nano and Biophotonics, Ghent University, Gent, Belgium \\ * To whom correspondence should be sent: \\ Jonathan.DeRoo@ugent.be and Zeger.Hens@ugent.be
}

Contact information of authors:

\author{
*Jonathan De Roo \\ E-mail: Jonathan.DeRoo@ugent.be
}

Address: Ghent University, Department of Inorganic and Physical Chemistry, Krijgslaan 281(S3), B-9000 Gent, Belgium

Isabel Van Driessche

E-mail: Isabel.VanDriessche@ugent.be 
Address: Ghent University, Department of Inorganic and Physical Chemistry, Krijgslaan 281(S3), B-9000 Gent, Belgium

José C. Martins

E-mail: Jose.Martins@ugent.be

Address: Ghent University, Department of Organic and Macromolecular Chemistry, Krijgslaan 281(S4), B-9000 Gent, Belgium

*Zeger Hens

E-mail: Zeger.Hens@ugent.be

Address: Ghent University, Department of Inorganic and Physical Chemistry, Krijgslaan 281(S3), B-9000 Gent, Belgium

No prior discussion with Nature Editors made 


\section{Introductory paragraph}

Surface chemistry is a key enabler for colloidal nanocrystal applications. In this respect, metal oxide nanocrystals (NCs) stand out from other NCs as carboxylic acid ligands adsorb on their surface by dissociation to carboxylates and protons, ${ }^{1,2}$ the latter proving essential in electron transfer reactions. ${ }^{3}$ Here, we show that this binding motif sets the stage for chemically driven ligand displacement where the binding of amines or alcohols to $\mathrm{HfO}_{2} \mathrm{NCs}$ is promoted by the conversion of a bound carboxylic acid into a non-coordinating amide or ester. Furthermore, the sustained ligand displacement, following the addition of excess carboxylic acid, provides a catalytic pathway for ester formation, whereas the addition of esters leads to NC-catalyzed transesterification. Since sustained, chemically driven ligand displacement leaves the NCs including their surface composition - unchanged and preserves colloidal stability, metal oxide nanocrystals are thus turned into effective nanocatalysts that bypass the tradeoff between colloidal stability and catalytic activity.

\section{Main text}

Over the last 20 years, colloidal nanocrystals have emerged as extremely versatile state of matter. The physical and chemical properties of these hybrid objects depend both on the inorganic core and the ligand-capped surface with the latter also determining the colloidal stability. Controlling the surface has proven indispensable for all NC applications, ranging from thermoelectrics ${ }^{4}$ and thin film electronics ${ }^{5,6}$ to electrochromic applications. ${ }^{7}$ The first step in surface manipulation is understanding the NC-ligand bond, and the Covalent Bond Classification (CBC) provides an apt theoretical framework for this purpose. ${ }^{8}$ Ligands are defined as L-, X- or Z-type depending on the number of electrons that the neutral ligand contributes to the NC-ligand bond (2, 1 or 0 respectively, see Fig. 1). L-type ligands are Lewis bases, Z-type ligands are Lewis acids, both coordinating to stoichiometric NCs and the respective binding motifs are $\mathrm{NC}(\mathrm{L})$ and $\mathrm{NC}(\mathrm{Z})$. X-type ligands are one electron donors in their neutral form and for example 
hydroxide, ${ }^{9}$ halides ${ }^{10}$ and carboxylates ${ }^{11}$ bind to surface cations in a $\mathrm{NC}\left(\mathrm{MX}_{\mathrm{n}}\right)$ binding motif. Only in the special case of metal oxide NCs, it was shown that carboxylic acids provide two Xtype moieties, retrieved as surface adsorbed protons and carboxylates and denoted as $\mathrm{NC}(\mathrm{X})_{2} \cdot{ }^{1,2}$ Charge balancing requirements restrict ligand exchange reactions in apolar solvents, in first instance, to $\mathrm{L}$-for-L and $\mathrm{X}$-for-X exchange processes (see Fig. 1). ${ }^{12}$ These processes are governed by adsorption enthalpy (L- and X-type) and/or acidity (X-type) differences, hampering exchange for weaker binding and/or less acidic ligands. More elaborate reactions involve L-type ligands that promote the displacement of Z-type cadmium carboxylate ${ }^{13}$ from CdSe NCs - note that $\mathrm{NC}\left(\mathrm{CdX}_{2}\right)=\mathrm{NC}(\mathrm{Z})-$ or the displacement of carboxylic acids ${ }^{2}-\mathrm{NC}(\mathrm{X})_{2}-$ from $\mathrm{HfO}_{2} \mathrm{NCs}_{\text {, }}$ see Fig. 1. These reactions are facilitated by an enthalpy gain since the leaving ligand forms a complex or ion pair with the L-type ligands. Nevertheless, these reactions still rely on a huge excess of new ligand and work best for strong Lewis bases such as amines, whereas obtaining nanocrystals stabilized by, for example, alcohols proved nearly impossible. ${ }^{13}$

Here we show that, in the case of $\mathrm{HfO}_{2} \mathrm{NCs}$, weakly binding ligands such as amines and alcohols chemically convert tightly bound carboxylic acids in non-coordinating amides or esters and thus promote acid/amine and acid/alcohol ligand displacement. Furthermore, the ester formation is sustained when the ligand shell is continuously replenished with carboxylic acids, a reaction not occurring in the absence of the NCs. We relate this unexpected colloidal nanocatalysis to the dissociative $\mathrm{NC}(\mathrm{X})_{2}$ binding motif of carboxylic acids to oxide $\mathrm{NCs}$ and we further underscore its potential by showing that colloidal $\mathrm{HfO}_{2} \mathrm{NCs}$ also catalyze transesterifications. While deemed promising, as it may combine the benefits of heterogeneous and homogeneous catalysis, ${ }^{14}$ colloidal nanocatalysis is often problematic since surface-adsorbed ligands, needed for colloidal stability, prevent reagents from reaching catalytically active surface sites. ${ }^{15}$ Hence the description of thiol ligands as a double-edge sword for catalytic CO oxidation by $\mathrm{Au}$ nanoclusters ${ }^{16}$ and a general preference for heterogeneous approaches using supported, 
ligand-stripped nanocrystals over colloidal nanocatalysis. ${ }^{15,17,18}$ By using reagents as ligands however, chemically driven ligand displacement bypasses this colloidal stability / catalytic activity conundrum, thus turning colloidal nanocrystals into effective nanocatalysts.

\section{Chemically Driven Ligand Displacement}

$\mathrm{HfO}_{2} \mathrm{NCs}$ were synthesized by a solvothermal process and subsequently capped with oleic acid to ensure colloidal stability, ${ }^{19}$ a process resulting in a ligand shell of oleates and protons as represented in Figure 2a. ${ }^{2}$ The NC-oleate binding was apparent from the broadened oleyl resonances in the ${ }^{1} \mathrm{H}$ NMR spectrum (Supplementary Fig. S1) of the dispersion in $o$ dichlorobenzene- $d_{4}(\mathrm{DCB})$. Note that the spectrum was acquired at $60{ }^{\circ} \mathrm{C}$ since the NCs are not dispersible in DCB at room temperature. Our general strategy to have carboxylic acids replaced by amines on $\mathrm{HfO}_{2} \mathrm{NCs}$ is illustrated in Figure 2a. Rather than a direct amine-for-acid exchange, which proved unfavorable, ${ }^{2}$ we envisaged a process where one amine equivalent converts one acid equivalent into an amide, which is released from the surface, allowing a second amine equivalent to bind to the uncovered surface site. A first indication of this process occurring is the appearance of (green) resonances pertaining to the octyl amide of oleic acid in the ${ }^{1} \mathrm{H}$ NMR spectrum of a mixture of oleic acid capped $\mathrm{HfO}_{2} \mathrm{NCs}$ and octylamine, kept at $130{ }^{\circ} \mathrm{C}$ (Fig. $2 \mathrm{~b}$, detailed assignment in Supplementary Fig. S2). The alkene resonance (5.5 ppm) comprises both a broad resonance of remaining bound oleic acid and a sharp resonance of the formed amide. The width and fine structure of the latter suggest that the amide does not coordinate to the NCs, a conclusion confirmed by positive (red) cross peaks in the NOESY spectrum (Fig. 2c). In contrast, the resonances of octylamine and the remaining oleic acid feature negative (dark-blue) nOe cross peaks, indicative of bound ligands. ${ }^{20}$ Hence, amide formation coincides with the development of a mixed ligand shell, comprising both oleic acid and octylamine. 
The same strategy applies to the replacement of carboxylic acids by alcohols via ester formation, Fig. 2d. Again, its success follows from the sharp (blue) ester resonances showing up in the ${ }^{1} \mathrm{H}$ NMR spectrum (Fig. 2e, detailed assignment in Supplementary Fig. S3) and the ethanol resonances featuring pronounced negative cross peaks in the NOESY spectrum (Supplementary Fig. S4). The DOSY spectrum (Fig. 2f) sheds more light on the dynamic nature of the NC-ligand bond. The diffusion coefficient of the remaining oleic acid is small $\left(36 \mu \mathrm{m}^{2} / \mathrm{s}\right)$, corresponding to a solvodynamic diameter of $9 \mathrm{~nm}(6.5 \mathrm{~nm}$ inorganic core $+2.5 \mathrm{~nm}$ organic ligand shell). Oleic acid is thus tightly bound to the NCs as opposed to ethanol (or octylamine), which exchanges rapidly between a free and a bound state as evidenced by the combination of its large diffusion coefficient $\left(1524 \mu \mathrm{m}^{2} / \mathrm{s}\right)$ and its negative nOe cross peak. ${ }^{20}$

In contrast to aggressive stripping reagents, ${ }^{21,22}$ ligand displacement by amide or ester formation is a rather gentle reaction. Since it was performed in-situ in the NMR spectrometer, the gradual change in ligand shell composition could be monitored (Fig. 3a). A conversion plateau is observed, indicating that this reaction is fully equilibrium controlled and thus reversible. However, the reaction proved kinetically impeded below $100{ }^{\circ} \mathrm{C}$, which makes for a ready tailoring of the ligand shell composition by adjusting the reaction time at $130{ }^{\circ} \mathrm{C}$ and subsequently locking that configuration at lower temperature, see Fig. 3b. Furthermore, the equilibrium could be manipulated to almost full displacement by, e.g., the addition of a larger excess of alcohol or amine (however still far below the quantities used in ref. 13 for the displacement of Z-type Cd-oleate from CdSe NCs, Supplementary Fig. S5). Interestingly, we found that, opposite from the original oleic-acid-only ligand shell, a mixed oleic acid / octylamine (or ethanol) capping, renders $\mathrm{HfO}_{2} \mathrm{NCs}$ dispersible in DCB also at room temperature, a result emphasizing the practical importance of ligand shells with a finely tuned composition.

\section{Nanocatalysis by Sustained Ligand Displacement}


Since esters are industrially important chemicals, the outcome of the chemically driven ligand displacement - esters and $\mathrm{HfO}_{2} \mathrm{NCs}$ with loosely bound alcohol ligands - inspired us to use this ligand displacement reaction for catalysis, Fig. 4a. After esterification, an adsorption site is left for the excess alcohol to bind. However, if both alcohol and carboxylic acid are present in excess, alcohol binding will be suppressed by carboxylic acid adsorption. This may lead to a sustained ester formation while the $\mathrm{HfO}_{2} \mathrm{NCs}$ maintain their carboxylic acid capping, i.e., a catalytic process. We thus added oleic acid and ethanol to oleic acid capped $\mathrm{HfO}_{2} \mathrm{NCs}$ and monitored the development of the ester resonances at $130{ }^{\circ} \mathrm{C}$, Fig. $4 \mathrm{~b}$. Initially, the ester resonances linearly increase with time (Fig 4c), the ethanol resonances decrease and the bound oleic acid resonances keep a steady intensity during the whole experiment (Supplementary Fig. S6 and S7, respectively). Since no ester was formed in a control experiment without $\mathrm{HfO}_{2} \mathrm{NCs}$ (Supplementary Fig. S8), this indicates that all free adsorption sites - created by ester formation - are immediately replenished by fresh oleic acid, restoring the surface composition of the catalyst. The overall activation energy of the reaction was estimated at approximately $88 \mathrm{~kJ} / \mathrm{mol}$ in the temperature interval $110-130{ }^{\circ} \mathrm{C}$ (see Fig. 4c and Supplementary Fig. S9). In addition, the esterification reaction was monitored using methanol and after three hours, equilibrium was reached with a catalytic turnover number of 3.4, further underpinning the catalytic nature of the process (Supplementary Fig. S10).

In a control experiment using $\mathrm{CdSe} \mathrm{NCs}$ - featuring a $\mathrm{NC}\left(\mathrm{CdX}_{2}\right)$ binding motif where $\mathrm{X}$-type carboxylates bind to excess surface $\mathrm{Cd}^{2+}$ without co-adsorption of protons - no ester formation was observed (Supplementary Fig. S11). ${ }^{13}$ This emphasizes the importance of the $\mathrm{NC}(\mathrm{X})_{2}$ binding motif and the underlying chemistry of the metal oxide surface - providing both acidic and basic adsorption sites - for catalysis. A tentative reaction pathway is represented in Fig. 4a, where the carbonyl carbon is rendered electrophilic by coordination of the carboxylate to hafnium and back-bonding of the surface proton. In addition, attraction of the alcohol proton by 
an oxygen anion may increase the alcohol's nucleophilicity. The presence of surface-adsorbed protons at the $\mathrm{HfO}_{2}$ surface makes for reaction conditions where, unlike oleate stabilized CdSe NCs, ester formation can proceed through water elimination. To our knowledge, there is no previous account of $\mathrm{HfO}_{2}$ as an esterification catalyst and in comparison with earlier reports on porous, single metal oxide catalysts, the operating temperature of colloidal $\mathrm{HfO}_{2} \mathrm{NCs}$ is much lower $\left(130^{\circ} \mathrm{C}\right.$ vs. $\left.300-450{ }^{\circ} \mathrm{C}\right){ }^{23}$ Other common (solid state) esterification catalysts, e.g., (doped) zeolites, ${ }^{24}$ ion exchange resins, ${ }^{24}$ sulfated zirconia, ${ }^{24}$ or sulfated tin oxide, ${ }^{25}$ are more complex in architecture, demand higher synthesis temperatures or activation treatments. In addition, the surface areas are rather small while the surface area of colloidal nanocrystals can easily be increased by decreasing the NC diameter. Single metal (hafnium) oxide NCs may thus open a new class of esterification catalysts.

\section{Transesterification Catalysis by $\mathrm{HfO}_{2}$ Nanocrystals}

Further exploring $\mathrm{HfO}_{2} \mathrm{NC}$ catalysis, we found that heating oleic acid capped $\mathrm{HfO}_{2} \mathrm{NCs}$ to 130 ${ }^{\circ} \mathrm{C}$ in the presence of ethyl acetate leads to the formation of ethanol and acetic acid, i.e., ester hydrolysis (Supplementary Fig. S12), which did not occur in the absence of the NCs (Supplementary Fig. S13). This effect is probably caused by the dissociative adsorption of small amounts of residual water as $\mathrm{H}^{+}$and $\mathrm{OH}^{-}$, again $\mathrm{NC}(\mathrm{X})_{2}$, on the metal oxide surface (Supplementary Fig. 14). More importantly, nothing prevents the thus formed acetic acid or alcohol in getting involved in ester formation with a second alcohol or acid respectively, i.e., transesterification, Fig. 5a. This is exactly what is observed when heating a mixture of oleic acid capped $\mathrm{HfO}_{2} \mathrm{NCs}$, ethyl acetate and methanol, where we notice the primary formation of methyl oleate and methyl acetate, as indicated in green and blue in the ${ }^{1} \mathrm{H}$ NMR spectrum (Fig. 5b). Residual resonances of ethyl acetate and methanol are also observed, as is the reaction byproduct; ethanol. Such assignments are corroborated by the ${ }^{1} \mathrm{H}-\left\{{ }^{13} \mathrm{C}\right\} \mathrm{HMBC}$ spectrum (Fig. 5c) 
In addition, $22 \%$ of the oleic acid is converted into ethyl oleate, quite consistent with the original 1:3 ratio of ethyl acetate and methanol, indicating about equal rate of esterification and transesterification. Since solid-state acid catalyzed transesterification usually demands higher temperatures, the observed simultaneous catalysis of esterification and transesterification reactions by $\mathrm{HfO}_{2} \mathrm{NCs}$ are an interesting feat for, e.g., biodiesel applications where cheap feed stocks contain both triglycerides and free fatty acids. ${ }^{26}$ Note that in this experiment the original ligand is consumed in the reaction, without being replenished from the reaction mixture. Eventually, oleic acid is almost completely converted to ester (see the alkene resonance at 5.4 ppm in Fig. 5b) and the NCs are stabilized by only a very small amount of residual oleic acid as recognized by weak negative nOe cross peaks in the NOESY spectrum, Fig. 5d. In addition, ethanol and methanol are dynamically interacting with the surface as evidenced by their negative nOe cross peaks.

\section{Addressing the Activity/Stability Tradeoff in Nanocatalysis}

Colloidal metal nanocrystals have attracted wide attention as nanocatalysts since they can show enhanced catalytic activity as compared to bulk materials, ${ }^{27}$ combine the advantages of homogeneous and heterogeneous catalysts ${ }^{14}$ and constitute model systems to obtain molecular insight in catalytic processes. ${ }^{18}$ For practical purposes however, the enhanced catalytic activity often conflicts with the need to ensure colloidal stability as capping ligands tend to block catalytically active surface sites. ${ }^{15,18,28,29}$ From this point of view, chemically driven ligand displacement not only unexpectedly turns metal oxide $\mathrm{NCs}$ like $\mathrm{HfO}_{2}$ into a useful colloidal nanocatalyst, it also shows how the colloidal stability/catalytic activity tradeoff can be overcome. By turning steric stabilizers into reagents and supplying them in excess, full access to the catalytic sites is warranted without compromising colloidal stability. Importantly, the example of transesterification catalysis already shows that this approach extends beyond reactions where the originally present ligand is the only reacting/stabilizing species and similar processes are 
conceivable that involve small molecules, stabilizing NC dispersion in polar media. Moreover, as the catalytic activity is linked to intrinsic properties of the $\mathrm{HfO}_{2} \mathrm{NC}$ surface - preserved during the catalytic reaction - this approach makes for a far more robust nanocatalyst than nanocrystals functionalized by catalytically active ligands, ${ }^{14,18,30}$ where the catalytic properties are lost upon ligand desorption.

Given the extensive range of metal oxides that can be synthesized as nanocrystals, the almost unlimited possibilities to tune their composition and the variety of ligands they support, chemically driven ligand displacement on metal oxide nanocrystals may offer a new route towards low-cost, durable and versatile catalytic processes. We thus conclude that the joint catalysis of esterification and transesterification by $\mathrm{HfO}_{2}$ nanocrystals merely constitutes a first unique example of a much wider range of catalytic processes and metal oxide nanocatalysts to be explored in the near future.

\section{References}

1 De Roo, J. et al. Unravelling the surface chemistry of metal oxide nanocrystals, the role of acids and bases. J. Am. Chem. Soc. 136, 9650-9657 (2014).

2 De Roo, J. et al. Carboxylic-acid-passivated metal oxide nanocrystals: Ligand exchange characteristics of a new binding motif. Angew. Chem.-Int. Edit. 54, 64886491 (2015).

3 Schrauben, J. N. et al. Titanium and zinc oxide nanoparticles are proton-coupled electron transfer agents. Science 336, 1298-1301 (2012).

4 Ibáñez, M. et al. Electron doping in bottom-up engineered thermoelectric nanomaterials through hcl-mediated ligand displacement. J. Am. Chem. Soc. 137, 4046-4049 (2015).

5 Tang, J. et al. Colloidal-quantum-dot photovoltaics using atomic-ligand passivation. Nat. Mater. 10, 765-771 (2011).

6 Lee, J. S., Kovalenko, M. V., Huang, J., Chung, D. S. \& Talapin, D. V. Band-like transport, high electron mobility and high photoconductivity in all-inorganic nanocrystal arrays. Nat. Nanotechnol. 6, 348-352 (2011).

7 Llordes, A., Garcia, G., Gazquez, J. \& Milliron, D. J. Tunable near-infrared and visiblelight transmittance in nanocrystal-in-glass composites. Nature 500, 323-326 (2013).

8 Green, M. L. H. \& Parkin, G. Application of the covalent bond classification method for the teaching of inorganic chemistry. J. Chem. Educ. 91, 807-816 (2014).

9 Zherebetskyy, D. et al. Hydroxylation of the surface of pbs nanocrystals passivated with oleic acid. Science 344, 1380-1384 (2014). 
10 Owen, J. S., Park, J., Trudeau, P. E. \& Alivisatos, A. P. Reaction chemistry and ligand exchange at cadmium-selenide nanocrystal surfaces. J. Am. Chem. Soc. 130, 1227912280 (2008).

11 Fritzinger, B., Capek, R. K., Lambert, K., Martins, J. C. \& Hens, Z. Utilizing selfexchange to address the binding of carboxylic acid ligands to cdse quantum dots. $J$. Am. Chem. Soc. 132, 10195-10201 (2010).

12 Dierick, R. et al. Surface chemistry of CuInS2 colloidal nanocrystals, tight binding of l-type ligands. Chem. Mat. 26, 5950-5957 (2014).

13 Anderson, N. C., Hendricks, M. P., Choi, J. J. \& Owen, J. S. Ligand exchange and the stoichiometry of metal chalcogenide nanocrystals: Spectroscopic observation of facile metal-carboxylate displacement and binding. J. Am. Chem. Soc. 135, 1853618548 (2013).

14 Shylesh, S., Schunemann, V. \& Thiel, W. R. Magnetically separable nanocatalysts: Bridges between homogeneous and heterogeneous catalysis. Angew. Chem.-Int. Edit. 49, 3428-3459 (2010).

15 Huang, W., Hua, Q. \& Cao, T. Influence and removal of capping ligands on catalytic colloidal nanoparticles. Catal Lett 144, 1355-1369 (2014).

$16 \mathrm{Wu}, \mathrm{Z}$. et al. Thiolate ligands as a double-edged sword for co oxidation on ceo 2 supported au25(sch2ch2ph)18 nanoclusters. J Am Chem Soc 136, 6111-6122 (2014).

17 Zeng, H. C. Integrated nanocatalysts. Accounts Chem. Res. 46, 226-235 (2013).

$18 \mathrm{Niu}, \mathrm{Z}$. \& Li, Y. Removal and utilization of capping agents in nanocatalysis. Chem. Mat. 26, 72-83 (2014).

19 De Roo, J. et al. Fast, microwave-assisted synthesis of monodisperse hfo 2 nanoparticles. J. Nanopart. Res. 15, 1778 (2013).

20 Hens, Z. \& Martins, J. C. A solution nmr toolbox for characterizing the surface chemistry of colloidal nanocrystals. Chem. Mat. 25, 1211-1221 (2013).

21 Rosen, E. L. et al. Exceptionally mild reactive stripping of native ligands from nanocrystal surfaces by using meerwein's salt. Angew. Chem.-Int. Edit. 51, 684-689 (2012).

22 Dong, A. G. et al. A generalized ligand-exchange strategy enabling sequential surface functionalization of colloidal nanocrystals. J. Am. Chem. Soc. 133, 998-1006 (2011).

23 McNeff, C. V. et al. A continuous catalytic system for biodiesel production. Appl. Catal. A-Gen. 343, 39-48 (2008).

24 Kiss, A. A., Dimian, A. C. \& Rothenberg, G. Solid acid catalysts for biodiesel production - towards sustainable energy. Adv. Synth. Catal. 348, 75-81 (2006).

25 José da Silva, M. \& Lemos Cardoso, A. Heterogeneous tin catalysts applied to the esterification and transesterification reactions. Journal of Catalysts 2013, 1-11 (2013).

26 Meher, L. C., Sagar, D. V. \& Naik, S. N. Technical aspects of biodiesel production by transesterification - a review. Renew. Sust. Energ. Rev. 10, 248-268 (2006).

27 Narayanan, R. \& El-Sayed, M. A. Catalysis with transition metal nanoparticles in colloidal solution: Nanoparticle shape dependence and stability. J. Phys. Chem. B 109, 12663-12676 (2005).

28 Chen, K., Wu, H. T., Hua, Q., Chang, S. J. \& Huang, W. X. Enhancing catalytic selectivity of supported metal nanoparticles with capping ligands. Phys. Chem. Chem. Phys. 15, 2273-2277 (2013). 
29 Jenkins, S. V., Chen, S. \& Chen, J. Gold-copper alloyed nanorods for metal-catalyzed organic reactions: Implication of surface ligands on nanoparticle-based heterogeneous catalysis. Tetrahedron Lett. 56, 3368-3372 (2015).

30 Cano, I. et al. Air-stable gold nanoparticles ligated by secondary phosphine oxides as catalyst for the chemoselective hydrogenation of substituted aldehydes: A remarkable ligand effect. J. Am. Chem. Soc. 137, 7718-7727 (2015).

\section{Methods}

Nanocrystal synthesis. $\mathrm{HfO}_{2} \mathrm{NCs}$ were synthesized via an established microwave-assisted solvothermal process with some slight modifications. ${ }^{19}$ The precursor preparation was executed in a nitrogen filled glovebox. Under vigorous stirring, $0.5 \mathrm{~mL}$ of dibenzyl ether was added to 0.4 mmol $(0.13 \mathrm{~g})$ of hafnium chloride in a $10 \mathrm{~mL}$ microwave vial. Then quickly $4 \mathrm{~mL}$ of benzyl alcohol is added, yielding a transparant solution after $5 \mathrm{~min}$ of stirring. The solution was subjected to microwave heating with the following temperature settings: 5 min at $60{ }^{\circ} \mathrm{C}$ and 3.5 hours at $210{ }^{\circ} \mathrm{C}$. After synthesis, the phase separated mixture is transferred to a plastic centrifugation tube and $3 \mathrm{~mL}$ of diethyl ether is added. After mild centrifugation $(2000 \mathrm{rpm}, 2$ min) two phases are observed. The transparent organic (top) phase is removed and ethanol is added to the aqueous (bottom) phase yielding $2 \mathrm{~mL}$ translucent suspension. If not, centrifuge again $2000 \mathrm{rpm}, 2 \mathrm{~min}$ to remove agglomerates. The particles are precipitated and washed once with diethyl ether, resulting in $\mathrm{HfO}_{2} \mathrm{NCs}$, capped with $0.05 \mathrm{mmol}$ of $\mathrm{HCl}^{1}$. Finally the particles are redispersed by sonication in chloroform and $60 \mu \mathrm{L}$ oleic acid $(0.19 \mathrm{mmol})$ is added to the milky suspension. Under stirring, $50 \mu \mathrm{L}$ oleylamine $(0.15 \mathrm{mmol})$ was added and instantly a transparent suspension is obtained. The particles are purified five times by adding acetone, followed by centrifugation and resuspension in chloroform. This yields a colloidally stable dispersion of $\mathrm{HfO}_{2} \mathrm{NCs}$, capped with dissociatively adsorbed oleic acid and free of chloride. ${ }^{1}$

Esterification catalysis. The $\mathrm{HfO}_{2} \mathrm{NC}$ dispersion in chloroform is evaporated and deuterated $o$ dichlorobenzene (DCB) is added. The total content of NCs is $21.8 \mathrm{mg}(0.104 \mathrm{mmol})$ in $500 \mu \mathrm{L}$ 
DCB. Although at room temperature, the oleic acid capped NCs are not colloidally stable in DCB, heating to $60{ }^{\circ} \mathrm{C}$ or more yields a colorless and transparent suspension. The warm suspension is transferred to a screw capped NMR tube and the concentration of bound oleic acid is determined to be $21.4 \mathrm{mmol} / \mathrm{L}$ (yielding a ligand density of $3 \mathrm{~nm}^{-2}$ for the NCs with a diameter of $6.5 \mathrm{~nm}$ and an acid loading of $0.5 \mathrm{mmol} / \mathrm{g})$. Ethanol $(4 \mu \mathrm{L})$ and oleic acid $(8 \mu \mathrm{L})$ are added and the final concentration, measured at $60{ }^{\circ} \mathrm{C}$, was $115 \mathrm{mmol} / \mathrm{L}$ ethanol and $70 \mathrm{mmol} / \mathrm{L} \mathrm{HOAc}$ (bound + excess). The temperature is subsequently raised in a stepwise manner to $130{ }^{\circ} \mathrm{C}$ and kept there for 3 hours while 1D spectra are continuously recorded.

Solution NMR Characterization: Nuclear Magnetic Resonance (NMR) measurements were recorded on a Bruker Avance II Spectrometer operating at a ${ }^{1} \mathrm{H}$ and ${ }^{13} \mathrm{C}$ frequency of 500.13 $\mathrm{MHz}$ and $125.77 \mathrm{MHz}$ respectively and featuring a ${ }^{1} \mathrm{H},{ }^{13} \mathrm{C},{ }^{31} \mathrm{P}$ TXI-Z probe. The sample temperature was set to $298.15 \mathrm{~K}$ unless otherwise stated. One dimensional (1D) ${ }^{1} \mathrm{H}$ and $2 \mathrm{D}{ }^{1} \mathrm{H}$ $\left\{{ }^{13} \mathrm{C}\right\}$ HSQC, 2D ${ }^{1} \mathrm{H}-\left\{{ }^{13} \mathrm{C}\right\}$ HMBC, TOCSY and NOESY (Nuclear Overhauser Effect Spectroscopy) spectra were acquired using standard pulse sequences from the Bruker library. For the quantitative $1 \mathrm{D}{ }^{1} \mathrm{H}$ measurements, $64 \mathrm{k}$ data points were sampled with the spectral width set to $16 \mathrm{ppm}$ and a relaxation delay of $30 \mathrm{~s}$. NOESY mixing time was set to $300 \mathrm{~ms}$ and 4096 data points in the direct dimension for 512 data points in the indirect dimension were typically sampled, with the spectral width set to $11.5 \mathrm{ppm}$. For 2D processing, the spectra were zero filled to a $4096 \times 2048$ real data matrix. Before Fourier transformation, the $2 \mathrm{D}$ spectra were multiplied with a squared cosine bell function in both dimensions, the 1D spectra were multiplied with an exponential window function. Concentrations were obtained using the Digital ERETIC method. Diffusion measurements (2D DOSY) were performed using a double stimulated echo sequence for convection compensation and with monopolar gradient pulses. Smoothed rectangle gradient pulse shapes were used throughout. The gradient strength was varied linearly from $2-95 \%$ of the probe's maximum value (calibrated at $50.2 \mathrm{G} / \mathrm{cm}$ ) in 64 steps, with the gradient pulse duration 
and diffusion delay optimized to ensure a final attenuation of the signal in the final increment of less than $10 \%$ relative to the first increment. 


\section{Acknowledgements}

This work was financially supported by the Research Foundation Flanders (FWO). We are thankful to prof. dr. Pascal Van Der Voort and dr. Els De Canck for scientific discussions regarding the catalytic properties. The NMR equipment used in this work was financed through a grant (AUGE09006) from the Hercules foundation. Z.H. acknowledges Ghent University for funding (GOA 01G01513).

\section{Author contributions}

J.D.R. designed and carried out the experiments, interpreted the data, made the figures and wrote the manuscript. I.V.D. and Z.H. initiated the research and supervised the experiments. Z.H. also wrote the manuscript. J.C.M. supervised the research, provided the NMR instrumentation and aided in interpretation and discussions regarding the NMR data. All authors commented on the manuscript.

\section{Competing financial interests}

The authors declare no competing financial interests. 


\section{Figure Legends}

Figure 1 | NC-Ligand classification and exchanges/displacements. L-type ligands are Lewis bases, donating 2 electrons to the NC-Ligand bond. Z-type ligands are Lewis acids, offering an empty orbital. X-type ligands offer 1 electron to the NC-ligand bond. L-for-L exchanges, X-for$\mathrm{X}$ exchanges and Z-type displacements are illustrated with the model system of CdSe but could be extended to other $\mathrm{NCs}$ such as $\mathrm{PbS}, \mathrm{PbSe}, \mathrm{CdS}, \mathrm{ZnS}$, etc. $\mathrm{NC}(\mathrm{X})_{2}$ displacements are only known for oxides, e.g., $\mathrm{HfO}_{2}$.

Figure 2 Ligand displacement via amide formation and esterification. a, Carboxylic acid ligands are converted to amides, leading to amine binding. b, ${ }^{1} \mathrm{H}$ NMR spectra of a $\mathrm{HfO}_{2} \mathrm{NC}$ suspension in $o$-dichlorobenzene- $d_{4}$, stabilized by oleic acid, before and after addition of 10 equivalents of octylamine and reaction at $130{ }^{\circ} \mathrm{C}$ for three hours. Spectra acquired at 60 and 130 ${ }^{\circ} \mathrm{C}$ respectively. c, Room temperature NOESY spectrum of the resulting suspension. Note how the $\mathrm{N}-\mathrm{H}$ resonance moved downfield due to the temperature change. d, Carboxylic acid ligands are converted to esters, leading to alcohol binding. e, ${ }^{1} \mathrm{H}$ NMR spectra of a $\mathrm{HfO}_{2} \mathrm{NC}$ suspension in $o$-dichlorobenzene- $d_{4}$, stabilized by oleic acid, before and after addition of 7 equivalents of ethanol and reaction at $130{ }^{\circ} \mathrm{C}$. Spectra acquired at 60 and $130{ }^{\circ} \mathrm{C}$ respectively. f, Room temperature DOSY spectrum of the resulting suspension. The alkene resonance of bound oleic acid is not observed due to the combination of its short $\mathrm{T}_{2}$ relaxation time, the length of the convection compensating pulse program and the large NC size.

Figure 3 | Ligand shell composition. a, The fraction of bound oleic acid decreases gradually with time until equilibrium when the $\mathrm{HfO}_{2} \mathrm{NC}$ suspension in $o$-dichlorobenzene- $d_{4}$ is heated with either 7 equivalents of ethanol or 10 equivalents of octylamine. $\mathbf{b}$, The fraction of bound oleic acid is decreasing at $130{ }^{\circ} \mathrm{C}$ due to amide formation with 8 equivalents of octylamine. However at $60{ }^{\circ} \mathrm{C}$, even before equilibrium is reached, that fraction remains constant, i.e. kinetic trapping of the ligand shell composition.

Figure 4 | Catalyzed esterification. a, Starting from pristine hafnium oxide NCs, the alcohol penetrates the ligand shell and reacts with the bound acid to form an ester. This leaves a free adsorption site which is subsequently occupied by a new carboxylic acid molecule and the catalytic cycle continues. b, NMR spectra in $o$-dichlorobenzene- $d_{4}$, acquired at different reaction times at $130{ }^{\circ} \mathrm{C}$ for the esterification of oleic acid and ethanol with $\mathrm{HfO}_{2} \mathrm{NCs}$ as catalyst. Ratio of $\mathrm{HOAc}$ and $\mathrm{EtOH}=1: 1.6$. c, The esterification rate at different temperatures.

Figure 5 | Catalyzed transesterification. a, Esters are converted in new esters by adding new alcohol and simultaneous with transesterification, esterification takes place. $\mathbf{b},{ }^{1} \mathrm{H}$ NMR spectrum acquired after 2 hours at $130{ }^{\circ} \mathrm{C}$ of a $\mathrm{HfO}_{2} \mathrm{NC}$ suspension in $o$-dichlorobenzene- $d_{4}$ with 5 equivalents of ethyl acetate and 15 equivalents of methanol (with respect to bound oleic acid). c, The HMBC spectrum, correlating the carbonyl carbons to neighbouring protons, confirmed the existence of 4 ester species in the synthesis mixture d, NOESY spectrum. Negative cross peak are observed for ethanol, methanol and oleic acid proving a bound state. The ester resonance only features zero quantum coherences. 


\section{Figures}

Figure 1

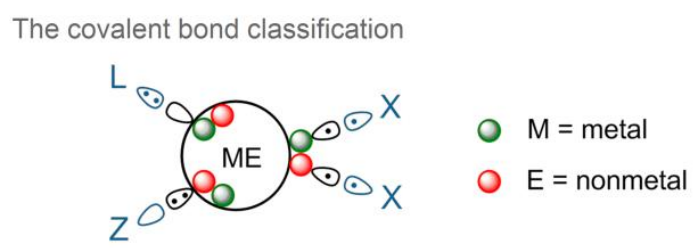

$L$ for $L$ exchange

Case ${ }_{-L}+L^{\prime} \longrightarrow$ CdSe $_{-L^{\prime}}+L$

$X$ for $X$ exchange

$$
\text { Cdse }<<X_{X}^{X}+H X^{\prime} \longrightarrow<-\frac{C^{\prime}}{X}+H X
$$

$\mathrm{NC}(\mathrm{Z})$ displacement

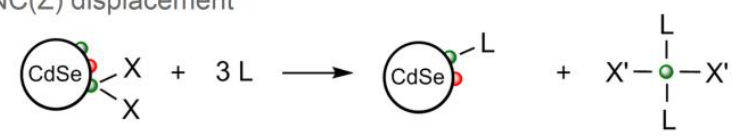

$\mathrm{NC}(\mathrm{X})_{2}$ displacement

$$
\longrightarrow \text { H }
$$

Figure 2

a

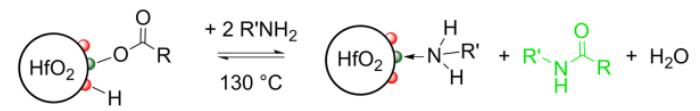

$$
\begin{aligned}
& \text { - hafnium } \bigcirc \text { oxygen }
\end{aligned}
$$

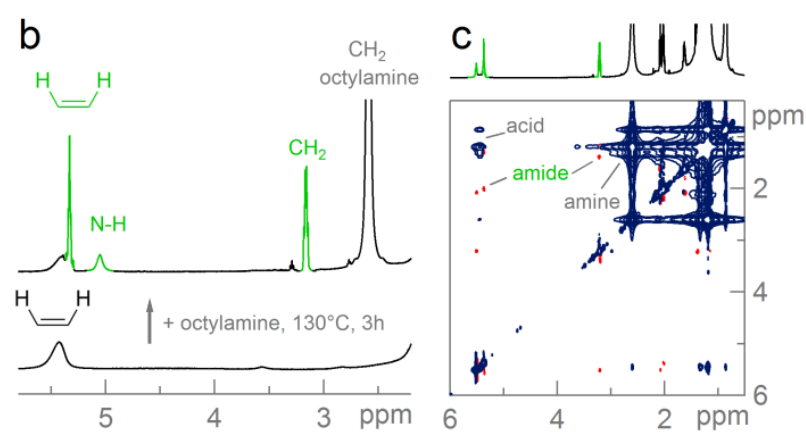

d

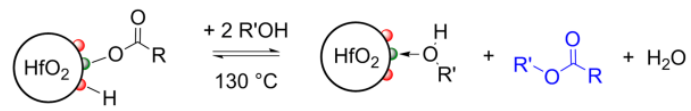

$$
\begin{aligned}
& \text { hafnium } \bigcirc \text { oxygen }
\end{aligned}
$$

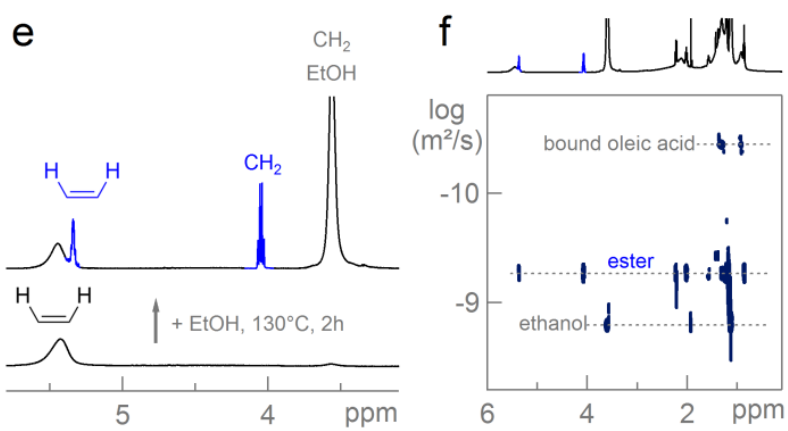


Figure 3

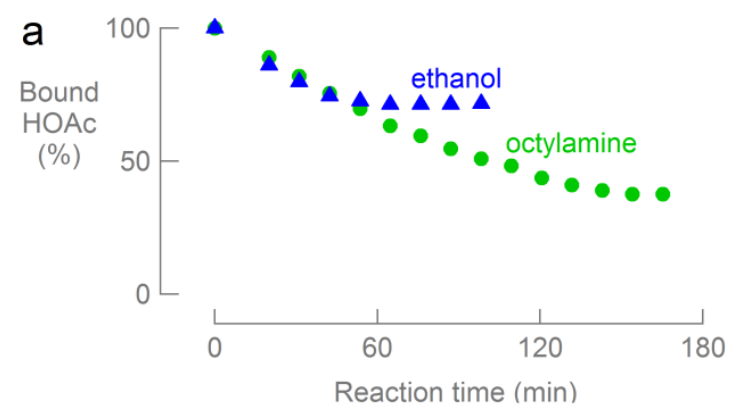

b

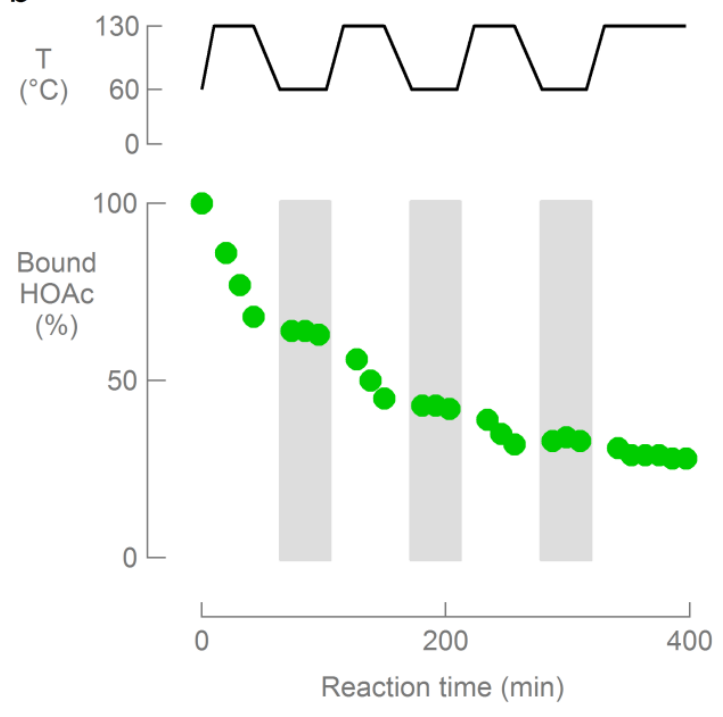


Figure 4

a
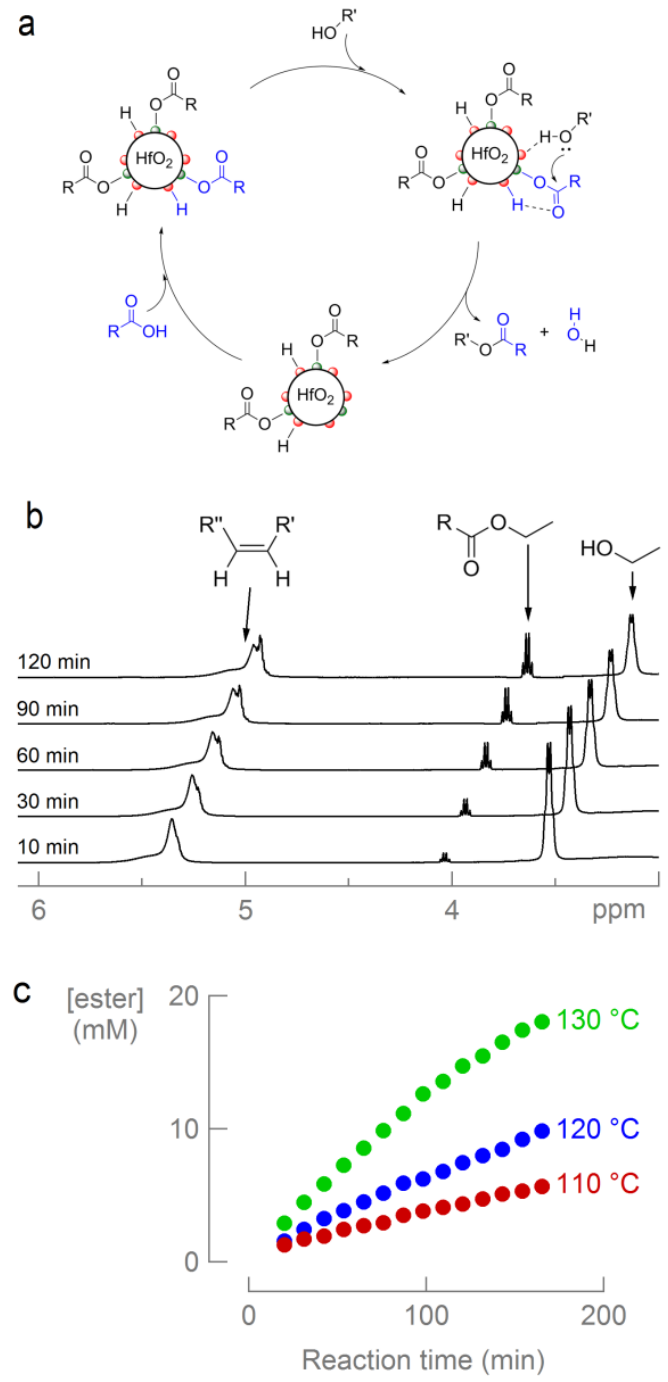
Figure 5

a

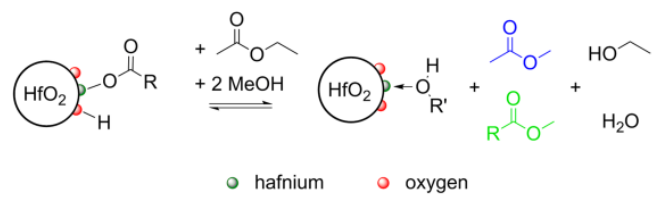
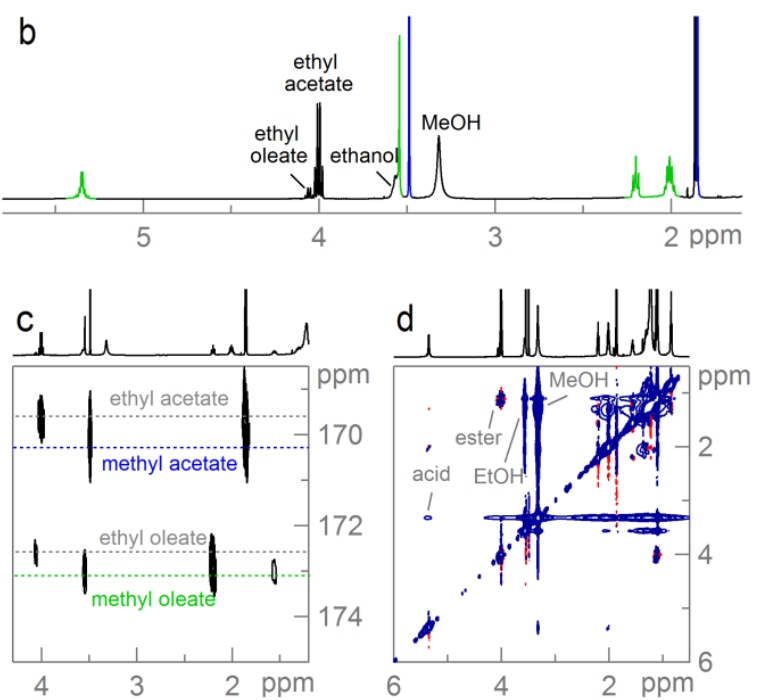
